\title{
Estudo comparativo in vitro do movimento de gaveta em joelhos de caninos submetidos a duas técnicas extracapsulares de correção da instabilidade após ruptura do ligamento cruzado cranial
}

\author{
In vitro comparative study of canine knees drawer movement submitted to two extra-capsular \\ techniques to fix the instability after abruption of the cranial cruciate ligament
}

\author{
Camila Fernandes Pelisson ${ }^{\mathrm{I}}$ Silvia Manduca Trapp ${ }^{\mathrm{II}}$ Bernardo Kemper ${ }^{\mathrm{II}}$
}

RESUMO

A ruptura do ligamento cruzado cranial (RLCCr) é uma desordem ortopédica comum em cães que promove a instabilidade da articulação do joelho, resultando em claudicação e alterações degenerativas progressivas desta. A melhor técnica para reparação da $R L C C r$ ainda não está bem elucidada. A maioria das técnicas alcança uma taxa de sucesso entre 80 a $90 \%$ e todas apresentam vantagens e desvantagens. $O$ objetivo deste trabalho foi avaliar os resultados da estabilização extracapsular utilizando dois pontos distintos de ancoragem na articulação femoro-tibio-patelar como forma de tratamento da RLCCr em cães. Para tanto, foram utilizados 20 membros pélvicos de caninos. Após a RLCCr, todos os membros receberam avaliação da eficiência da técnica por meio do estudo da estabilidade crânio-caudal do joelho em flexão de $90^{\circ}$ e extensão de $135^{\circ}$. As estabilizações extracapsulares realizadas nos cadáveres conferiram estabilidade crânio-caudal sem demonstrar superioridade de uma ou outra técnica, e nenhuma delas pode atribuir estabilidade isométrica quando testadas com os membros em $90^{\circ}$ e $130^{\circ}$.

Palavras-chave: articulação, ponto isométrico, tratamento cirúrgico.

\section{ABSTRACT}

The rupture of the cranial cruciate ligament (RLCCr) is a common orthopedic disorder in dogs resulting in instability of the knee that promotes: lameness and progressive degenerative changes. The best technique to repair RLCCr, is not well elucidated. Most techniques achieve a success rate between 80 to $90 \%$ and all have advantages and disadvantages. The objective of this study was to evaluate the results of extracapsular stabilization using two different points of anchorage in the femora tibio-patellar joint, as a way of repairing of
RCCrL in dogs, which were used for 20 members of pelvic canines. After RCCrL all members were assessed to verify the efficiency of the technique through the study of cranial caudal stability of the knee in $90^{\circ}$ of flexion and extension of $130^{\circ}$. The extra-capsular stabilization performed in cadavers brought stability without demonstrating superiority of either technique, and both could not give isometric stability when tested with the members positioning in the angle of $90^{\circ}$ and $130^{\circ}$.

Key words:. knuckle, isometric point, surgical technique.

\section{INTRODUÇÃO}

O joelho é uma complexa articulação ginglimoide composta por duas articulações (femoropatelar e femorotibial) funcionalmente distintas, e a articulação femoropatelar aumenta muito a eficácia mecânica do grupo muscular do quadríceps e facilita a função dos extensores. Há também quatro ossos sesamoides: a patela, a fabela medial e lateral e o sesamoide poplíteo (VASSEUR, 1998; TOMLINSON, 2001).

O ligamento cruzado cranial (LCCr) se divide em faixas craniomedial e caudolateral, que apresentam pontos de inserção diferentes no platô tibial. A faixa craniomedial é estirada durante todas as fases de flexão e extensão, enquanto a faixa caudolateral fica estirada apenas em extensão e torna-se frouxa em flexão. O LCCr também atua limitando a rotação interna da tíbia em relação ao fêmur (SCHULZ, 2007) e ainda impede o

IMédica Veterinária Autônoma, Ibiporã, PR, Brasil.

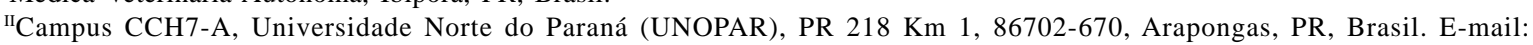
bkemper@bol.com.br. *Autor para correspondência. 
deslocamento cranial da tíbia (PIERMATTEI et al., 2006; SCHULZ, 2007).

A RLCCr é uma das anormalidades ortopédicas mais comumente encontradas nos joelhos dos cães (BUQUERA et al., 2002; HAYASHI et al., 2003; PIERMATTEI et al., 2006). Essa ruptura ligamentar pode ser completa ou parcial (SCHULZ, 2007), apresentando instabilidade variada, e em ambos os casos os animais não tratados desenvolverão doença articular degenerativa (DAD) (VASSEUR, 1998; PIERMATTEI et al., 2006). A RLCCr pode afetar qualquer sexo, idade ou raça de cães; no entanto, a maioria dos cães acometidos são de raças grandes, jovens e ativos (SCHULZ, 2007).

A confirmação clínica de RLCCr se dá pela presença do movimento de gaveta que causa deslizamento da tíbia cranial ou caudalmente em relação ao fêmur (SCHULZ, 2007). O teste é considerado positivo quando o deslocamento crânio-caudal da tíbia em relação ao fêmur é superior a $2 \mathrm{~mm}$, podendo atingir $8 \mathrm{~mm}$ ou mais nos casos de maior instabilidade (MOORE \& READ 1996).

O mecanismo da lesão traumática do LCCr é primariamente reflexo da sua função de contenção dos movimentos articulares (SCHULZ, 2007). A causa da lesão é principalmente o constante estresse transmitido através do ligamento e de forças excessivas durante extremos de movimentação (ARNOCZKY, 1996b; VASSEUR, 1998; ROOSTER et al., 2006).

A degeneração do ligamento, juntamente com atividades normais repetitivas, pode causar a ruptura progressiva deste, estando frequentemente associada a anormalidades conformacionais dos membros (ARNOCZKY, 1996b; VASSEUR, 1998) e ao envelhecimento (VASSEUR, 1998; SCHULZ, 2007), levando à alteração do colágeno e resultando em perda de tensão e rigidez. Além disso, são observadas tensões excessivas na estrutura do ligamento (VASSEUR, 1998) e artropatias imunomediadas, em ambos os joelhos. Muitos animais apresentam RLCCr bilateral ou do ligamento contralateral de um a dois anos após a ruptura do primeiro (SCHULZ, 2007).

A decisão da terapia mais adequada em cães com RLCCr é influenciada pela idade, pelo porte e pelo peso do animal, pelo seu uso pretendido, por afecções ortopédicas ou clínicas concomitantes, pelas considerações econômicas e pela antecipada cooperação do dono do animal (VASSEUR, 1998; PIERMATTEI, et al., 2006). O alívio da dor, desconforto articular, melhoramento no funcionamento da articulação e controle da progressão da afecção são alguns dos princípios básicos do tratamento que se aplica à DAD (BEALE \& GORING 1996; PIERMATTEI, et al., 2006).
A terapia conservadora consiste, basicamente, em restrição da atividade física, caminhadas com coleira, uso de analgésicos e fisioterapia conforme a necessidade (VASSEUR, 1998), além da aplicação de bandagem conforme indicada por PIERMATTEI, et al. (2006), para cães de pequeno porte (até $10 \mathrm{~kg}$ ). Cães entre 15 e $20 \mathrm{~kg}$ apresentam evolução contínua dos sinais clínicos e osteoartrose de forma gradativa com o tratamento conservador (VASSEUR, 1998; DENNY \& BUTTERWORTH, 2006; SCHULZ, 2007).

O reparo do LCCr tem como principio básico o reestabelecimento da estabilidade articular (ARNOCZKY, 1996a) e deve mimetizar o mais próximo possível a cinemática normal do joelho (ROOSTER et al., 2006).

Para reparo cirúrgico dessa afecção, muitas técnicas foram descritas, entre elas, técnicas de reconstrução intra-articular, incluindo a utilização de materiais biológicos ou sintéticos ou mesmo a realização do reparo primário do ligamento com associação de outros materiais; as extra-articulares ou extracapsulares (TOMLINSON, 2001); e as osteotomias tibiais (KIM et al., 2008). Contudo, não foi determinada uma técnica cirúrgica que apresente resultado superior a todas as outras técnicas (LAZAR et al., 2005). Assim, a escolha do método utilizado depende do cirurgião e de sua aptidão (WARZEE et al., 2001; PIERMATTEI, et al., 2006; SCHULZ, 2007), levando-se em consideração critérios como tamanho, idade, função do cão e cronicidade da lesão (TOMLINSON, 2001).

CONZEMIUS et al. (2005) avaliou 131 cães da raça Labrador e três técnicas para correção da instabilidade da RLCCr, sendo elas: osteotomia niveladora do platô tibial (ONPT), transposição da fíbula e sutura fabelo-tibial. Nesse estudo, os melhores resultados clínicos foram obtidos com a técnica extracapsular e a ONPT.

Os procedimentos extracapsulares são tecnicamente mais facilmente realizáveis, portanto mais rápidos que os procedimentos intra-articulares. As técnicas extracapsulares têm como vantagens a redução do tempo cirúrgico, minimização de invasão articular, facilidade técnica e melhor resposta clínica. As técnicas mais empregadas são as de aplicação de fios monofilamentados de náilon colocados sobre a face lateral da articulação do joelho (SMITH, 2000), podendo-se empregar uma âncora no côndilo lateral para servir como ponto de fixação (SCHULZ, 2007).

A sutura fabelo-tibial utilizando fios de náilon é a técnica menos exigente, por ter uma manipulação e uma exposição tecidual mínima. Apesar de muito vantajosa, essa técnica extracapsular 
apresenta pequenas desvantagens, como compressão excessiva das superfícies articulares, soltura, afrouxamento ou rompimento do náilon (SMITH, 2000). O objetivo deste trabalho foi avaliar o efeito de duas técnicas extracapsulares sobre a estabilização da articulação do joelho, por meio da aferição do movimento de gaveta em joelhos de caninos, em flexão de $90^{\circ}$ e extensão de $130^{\circ}$, após a ruptura do LCCr.

\section{MATERIAL E MÉTODOS}

Para o desenvolvimento deste experimento, foram utilizados 20 membros pélvicos de cadáveres de caninos, com peso entre 4,5 e $12 \mathrm{~kg}$, provenientes do setor de patologia da Universidade Norte do Paraná (UNOPAR), situado no Campus de Arapongas, Paraná (PR). A escolha dos animais foi fundamentada no tamanho e peso do indivíduo, para a obtenção de um grupo homogêneo seguindo essas medidas. Em todos os joelhos, foi realizado exame ortopédico prévio para a eliminação de artropatias pré-existentes. Os membros foram tricotomizados da região lateral e medial da articulação coxofemoral até a articulação do tarso. Em seguida, foi realizada a artrotomia do joelho baseada na técnica de Paatsama descrita por PIERMATTEI \& GREELEY (2004).
Em todos os joelhos, o LCCr foi identificado e seccionado com o auxílio de uma lâmina de bisturi número 15 e confirmada a ruptura visualmente e clinicamente por meio do teste do movimento de gaveta. Após inspeção do espaço articular, realizou-se a síntese da cápsula articular com pontos isolados simples com fio náilon 2-0.

No experimento, foram comparadas duas técnicas de estabilização extracapsular do LCCr. A técnica A foi realizada conforme metodologia descrita por JOHNSON \& DUNNING (2005), sem a colocação de âncora, e a técnica B, através da colocação de âncora, segundo descrito por BEALE \& HULSE (2007). Em ambas, foram feitas medidas do movimento de gaveta, posicionando os joelhos em angulações de flexão de $90^{\circ}$ e extensão de $130^{\circ}$, sendo as angulações mensuradas com o auxílio de um goniômetro (CARCISão Paulo/SP).

Na realização da técnica $A$, identificou se a fabela lateral por palpação. Um fio multifilamentar de 050mm (Dyneema) foi transpassado com o auxílio de uma agulha (1/2 circulo, 30mm) ao redor desta (Figura 1, F1) em sua região medial, no sentido proximal à distal. Em seguida, com o auxílio de uma broca ortopédica de $2 \mathrm{~mm}$, fez-se um orifício na crista tibial (Figura 1, T1). O fio foi conduzido até a perfuração e, com o auxílio de

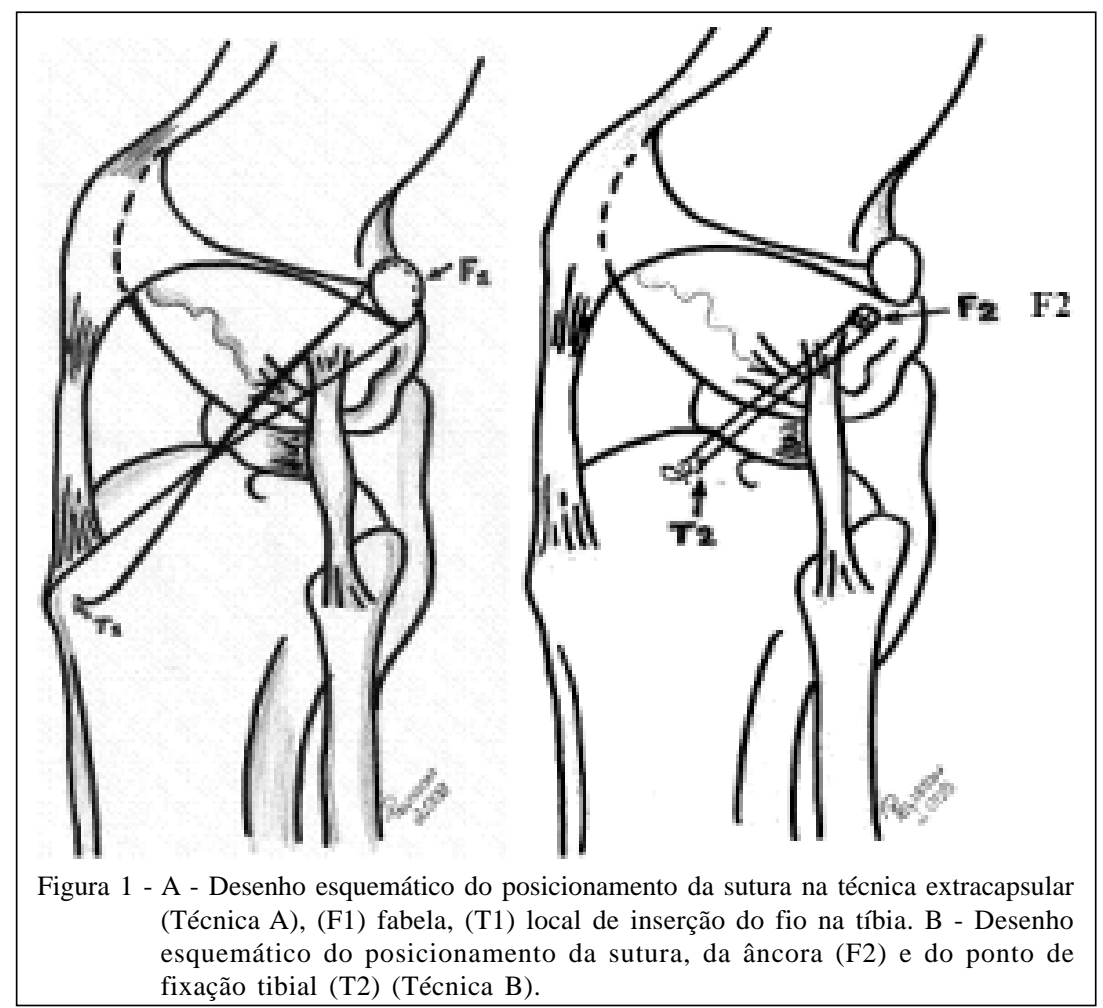

Ciência Rural, v.40, n.6, jun, 2010. 
uma agulha (40x12), foi transpassado no sentido lateral para medial e de volta por cima da crista tibial. Com o joelho flexionado na posição anatômica, realizou-se a amarração do fio, com nó de cirurgião duplo seguido de quatro nós simples (Figura 1).

Na técnica B, a fabela foi localizada por palpação. Uma perfuração foi feita com broca ortopédica de $2 \mathrm{~mm}$ e perfuratriz (150rpm) na região adjacente ao pólo distal à fabela, localizado sobre a origem do ligamento colateral lateral. Nesse ponto, uma âncora de 2,7x10mm (Bioconect-Itapira-SP) foi inserida (Figura 1, F2) com o auxílio de uma chave própria para âncoras (Bioconect-Itapira-SP). Outro orifício foi feito na porção proximal da tíbia cranialmente ao sulco do tendão extensor digital longo utilizando-se a mesma broca. Utilizou-se um fio com as mesmas características da técnica A. Assim, uma das extremidades do fio foi passada pelo orifício da âncora. Essa mesma extremidade foi transpassada pelo orifício da tíbia no sentido lateral para medial e colocou-se uma cavilha fixada na face medial do osso. Novamente o fio foi passado por esse mesmo orifício, mas em sentido oposto ao anterior com a finalidade de fixar a cavilha. $\mathrm{O}$ membro foi posicionado anatomicamente e então um nó de cirurgião simples foi feito no fio seguido de quatro nós simples (Figura 2).

Em todos os joelhos utilizados, fixou-se um pino (Kirschner 1,0mm) na porção proximal e outro na distal da inserção do ligamento colateral lateral, sem que dificultasse a colocação e o deslocamento do fio, que serviram de referência para medir o deslocamento da tíbia em relação ao fêmur durante o movimento de gaveta.

Após a realização de cada técnica, os membros foram posicionados lateralmente à superfície de uma mesa plana e, com o auxílio do goniômetro, o membro foi flexionado em $90^{\circ}$ e depois estendido em $130^{\circ}$, e o movimento de gaveta cranial da tíbia em relação ao fêmur foi testado para se verificar o grau de deslocamento. Todas as medições foram realizadas pelo mesmo avaliador com auxílio de paquímetro.

\section{RESULTADOS E DISCUSSÃO}

Muitos estudos enfocam o LCCr por ser o ligamento mais importante na estabilidade do joelho e também o mais vulnerável à ruptura (ROOSTER et al., 2006). Para o tratamento da RLCCr, varias técnicas cirúrgicas têm sido descritas (CONZEMIUS et al., 2005). A sutura ancorada na fabela e na crista tibial tem sido usada com sucesso há muito tempo para reduzir o deslocamento cranial da tíbia em relação ao fêmur após ruptura do LCCr (ROE et al., 2008). Essa técnica está associada a menos complicações trans e pósoperatórias, quando comparada a outras técnicas (CASALE et al., 2009).

A avaliação do deslocamento cranial da tíbia em relação ao fêmur confirma a ruptura do ligamento e avalia a sua instabilidade (SCHULZ, 2007). Um deslocamento crânio caudal de até $2 \mathrm{~mm}$ é considerado normal. Entretanto, o diagnóstico da RLCCr deve ser realizado de forma cuidadosa em cães jovens, nos quais

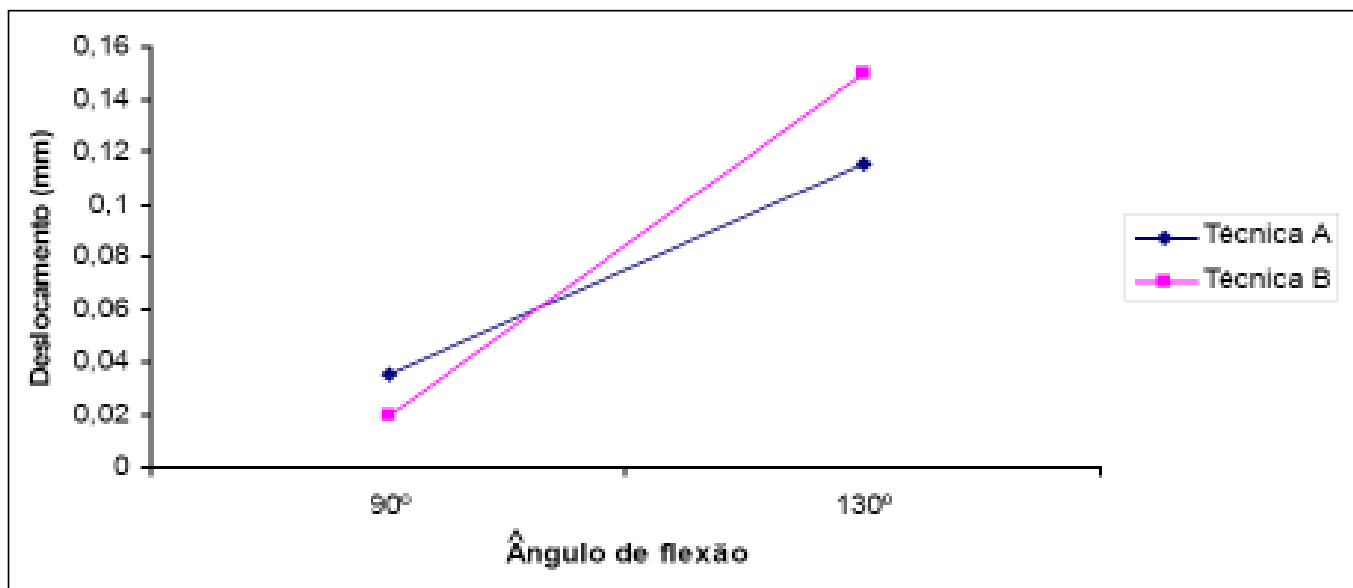

Figura 2 - Deslocamento crânio-caudal da tíbia em relação ao fêmur sob força de tração após as estabilizações extracapsulares (técnica A e B nas angulações de $90^{\circ}$ e $130^{\circ}$ ). Medidas em mm.

Ciência Rural, v.40, n.6, jun, 2010. 
o deslocamento crânio caudal da tíbia pode atingir até 4mm em articulações normais (MOORE \& RED, 1996).

Durante a flexão e extensão, os diferentes pontos de ancoragem se aproximam um do outro, e a sutura torna-se frouxa (ROE et al., 2008). Esse conhecimento motivou a realização de estudos in vitro para determinar o melhor posicionamento da fixação extracapsular nas diferentes escalas de flexão do joelho, tanto em cães (ROE et al., 2008), como em humanos (ZAVRAS, et al. 2005). Neste estudo em questão, comparou-se o efeito de duas técnicas extracapsulares sobre o movimento de gaveta em flexão de $90^{\circ}$ e extensão de $130^{\circ}$.

A média do deslocamento crânio-caudal da tíbia na angulação de $90^{\circ}$ na técnica A foi de $0,35 \mathrm{~mm}$, já o deslocamento observado na técnica $B$ foi de $0,20 \mathrm{~mm}$, o que demonstra que ambas as técnicas conferem estabilidade crânio-caudal ao joelho quando avaliadas nessa angulação. Mesmo que a técnica A tenha se mostrado com uma instabilidade levemente superior à técnica B, esse resultado se mostrou estatisticamente insignificante. Segundo MOORE \& RED (1995), uma pequena translação é palpável em alguns pontos após o procedimento em animais. Em um estudo de pontos isométricos, a localização da ancoragem caudal à fabela demonstrou-se o ponto ideal para a fixação femoral (ROE et al., 2008). Esse ponto foi utilizado na técnica B utilizando-se uma âncora para a fixação da sutura, o que poderia justificar a maior estabilidade promovida pela técnica nos joelhos avaliados. A avaliação da translação tibial foi feita uma única vez, porém estudos demonstraram que, em avaliações in vitro após 10 ciclos, um afrouxamento maior é observado utilizando nós (VIANNA \& ROE, 2006), repetições não realizadas neste estudo. Na figura 2, são representados os valores do deslocamento da articulação femoro-tíbio-patelar sob força de tração nas angulações de $90^{\circ}$ e $130^{\circ}$. Por meio da análise da tabela, observa-se a variação dos valores do deslocamento crânio-caudal da tíbia em relação ao fêmur, sob força de tração após as estabilizações extracapsulares.

Na avaliação do deslocamento crâniocaudal da tíbia com o joelho em extensão $\left(130^{\circ}\right)$, a média do deslocamento foi de $1,15 \mathrm{~mm}$ na técnica $\mathrm{A}$, e na técnica $B$ foi de $1,50 \mathrm{~mm}$, sendo a diferença entre elas estatisticamente insignificante. Porém, ambas promoveram a estabilidade do joelho após ruptura do LCCr. Quando comparadas as avaliações realizadas em extensão com aquelas do joelho em flexão, um maior movimento foi observado na extensão. Isso que indica que nenhuma das técnicas usou pontos verdadeiramente isométricos, como descritos por ROE et al., (2008). Na análise estatística desses resultados, não há diferença significativa entre as técnicas quando a força de tração foi aplicada.

A média, o desvio padrão e os intervalos máximo e mínimo das medidas no deslocamento crâniocaudal da tíbia em relação ao fêmur, após as estabilizações extracapsulares (técnica A e B nas angulações de $90^{\circ}$ e $130^{\circ}$ ), estão representados na tabela 1.

\section{CONCLUSÃO}

A estabilização extracapsular com as técnicas descritas nos cadáveres deste estudo conferiu estabilidade crânio-caudal sem demonstrar superioridade de uma em relação à outra. Em ambas, não foi possível atribuir estabilidade isométrica nas duas angulações testadas. Para validar os resultados encontrados, há a necessidade de realização de estudos clínicos in vivo.

Tabela 1 - Média, desvio padrão e intervalos máximo e mínimo das medidas no deslocamento crânio-caudal da tíbia em relação ao fêmur após as estabilizações extracapsulares (técnica A e B nas angulações de $90^{\circ}$ e $130^{\circ}$ ). Medidas em mm.

\begin{tabular}{lcccc}
\hline Angulo de flexão & $90^{\circ}$ & $90^{\circ}$ & \multicolumn{1}{c}{$130^{\circ}$} & $130^{\circ}$ \\
\hline Técnica & A & B & A & $1,150^{*}$ \\
Média & $0,350^{*}$ & $0,200^{*}$ & 1,040 & $1,500^{*}$ \\
Desvio & 0,590 & 0,410 & 4,000 & 3,000 \\
Intervalo & 2,000 & 1,000 & 0,000 & 0,000 \\
Min & 0,000 & 0,000 & 4,000 & 3,000 \\
Máx & 2,000 & 1,000 & & \\
\hline
\end{tabular}

Símbolos iguais nas colunas, na mesma angulação, indicam não haver diferença estatística com nível de significância (0,05 ou 5\%). 


\section{REFERÊNCIAS}

ARNOCZKY, S.P. Patomecânica das lesões do ligamento cruzado e meniscos. In: BOJRAB, M.J. Mecanismo da moléstia de pequenos animais. 2.ed. São Paulo: Manole, 1996a. Cap.110, p.889-902.

ARNOCZKY, S.P. Reparo do ligamento cruzado cranial. In: BOJRAB, M.J. Técnicas atuais em cirurgia de pequenos animais. 2.ed. São Paulo: Roca, 1996b. Cap.46, p.664-669.

BEALE, B, GORING, R. Artropatias imunomediadas. In: BOJRAB, M.J. Mecanismo da moléstia de pequenos animais. 2.ed. São Paulo: Manole, 1996. Cap.108, p.864881.

BEALE, B.S., HULSE, D.A. Canine cranial cruciate ligament repair anchor system canine cranial cruciate ligament repair anchor system. Arthrex Vet Systems. Disponível na Internet: www.arthrexvetsystems.com. Online. Acesso em: 2007.

BUQUERA, L.E.C. et al. Avaliação clínica do joelho após estabilização extra-articular utilizando fáscia lata, fio de poliéster ou fio de náilon na ruptura do ligamento cruzado cranial em cães. Veterinária Noticias, Uberlandia v.8, n.1, p.27-34, 2002.

CASALE, S.A.; MCCARTHY, R.J. Complications associated with lateral fabellotibial suture surgety for cranial cruciate ligament injury in dogs: 363 cases (1997-2005). Journal of the American Veterinary Medical Association, Schaumburg, v.234, n.2, p.229-235, 2009.

CONZEMIUS, M.G. et al. Effect of surgical technique on limb function after surgery for rupture of the cranial cruciate ligament in dog. Journal of the American Veterinary Medical Association, Schaumburg, v.226, n.2, p.232-236, 2005.

DENNY, H.R.; BUTTERWORTH, S.J. Cirurgia ortopédica em cães e gatos. 4.ed. São Paulo: ROCA, 2006. Cap.42, p.441-419.

HAYASHI, K. et al. Histologic changes in ruptured canine cranial cruciate ligament. Veterinary Surgery, Philadelphia, v.32, n.3, p.269-277, 2003.

JOHNSON, A.L.; DUNNING, D. Atlas of orthopedic surgical procedures of the dog and cat. St. Louis: Saunders, 2005. p.264.

KIM, S.E. et al. Tibial osteotomies for cranial cruciate ligament in dogs. Veterinary Surgery, Philadelphia, v.37, n.2, p.111125, 2008.

LAZAR, T.P et al. Long-term radiographic comparison of tibial plateau leveling osteotomy versus extracapsular stabilization for cranial cruciate ligament rupture in the dog. Veterinary Surgery, Philadelphia, v.34, p.133-141, 2005.
MOORE, K.W.; READ, R.A. Cranial cruciate ligament rupture in the dog a retrospective study comparing surgical techniques. Australian Veterinary Journal, Sydney, v.72, n.1, p.281285, 1995.

MOORE, K.W., READ, R.A. Rupture of cranial cruciate ligament in dogs-Part II. Diagnosis and management. Compendium on Continuing Education for the Practicing Veterinarian, Yardley, PA v.18, n.4, p.381-391, 1996.

PIERMATTEI, D.L. et al. The stifle joint. In: Handbook of small animal-orthopedics and fracture repair. 4.ed. Philadelphia: Saunders, 2006. p.818.

PIERMATTEI, D.L.; GREELEY, R.G. Atlas de abordagens cirúrgicas aos ossos do cão e gato. São Paulo: Manole, 2004. p.197.

ROE, S.C. et al. Isometric potential suture attachment sites for the cranial cruciate ligament deficient canine stifle. Veterinary and comparative orthopedics and traumatology, Stuttgart v.21, n.3, p.215-220, 2008.

ROOSTER, H. et al. Morphologic and functional Fratures of the canine cruciate ligaments. Veterinary Surgery, Philadelphia, v.35, n.8, p.769-780, 2006.

SCHULZ, K. Cranial cruciate ligament rupture. In: FOSSUM, T.W. Manual of small animal surgery. 3.ed. St. Louis: Mosby, 2007. Cap.33, p.1254-1275.

SMITH, B. Viewpoints in surgery cruciate ligament rupture, extracapsular stabilization. Australian Veterinary Journal, Sydney v.78, n.6, p.382-383, 2000.

TOMLINSON, J. Traditional repair techniques for the cranial cruciate deficiet stifle. In: WORLD SMALL ANIMAL VETERINARY, ASSOCIATION, 2001, Vancouver. Proceedings... Vancouver: WSAVA 2001.

VASSEUR, P.B. Articulação do joelho. In: SLATTER, D. Manual de cirurgia de pequenos animais. 2.ed. São Paulo: Manole, 1998. V.2, cap.137, p.2149-2180.

VIANNA, M.L.; ROE, S.C. Mechanical comparison of two knots and two crimp systems for securing nylon line used for extra-articular stabilization of the canine stifle. Veterinary Surgery, Philadelphia, v.35, n.6, p.567-572, 2006.

WARZEE, C.C. et al. Effect of the tibial plateau leveling on cranial and caudal tibial thrust in canine cranial cruciate deficient stifles: an in vitro experimental study. Veterinary Surgery, Philadelphia v.30, p.278-286, 2001.

ZAVRAS, T.D. et al. The effect of femoral attachment location on anterior cruciate ligament reconstruction: graft tension patterns and restoration of normal anterior-posterior laxity patterns. Knee Surgery, Sports Traumatology, Arthroscopy, Heidelberg, v.13, n. 2, p.92-100, 2005. 